\title{
State of The Art of Magnetic Resonance Imaging (MRI) in Evaluation of Focal Hepatic Lesions
}

\author{
Ahmed F. Youssef, Amany M. Elkharbotly, Hamada M. Khater, Magdy A. Awad
}

\begin{abstract}
Department of Radio diagnosis and Medical Imaging, Faculty of Medicine Benha University, Egypt.
\end{abstract}

Correspondence to: Magdy A. Awad, Department of Radio diagnosis and Medical Imaging, Faculty of Medicine Benha University, Egypt.

Email:

magdyawadbenha@gmail.com

Received: 12 November 2020

Accepted: 31 August 2021

\begin{abstract}
Background: The recent developments in MRI sequences for the upper abdomen comprise unenhanced diffusion-weighted imaging (DWI), and dynamic contrast-enhanced (DCE) MRI. DWI allows improved detection of small $(<10 \mathrm{~mm})$ focal liver lesions and it is useful as a road map sequence. The aim of this work was to study recent and advanced MRI techniques in the evaluation of focal hepatic lesions. Methods: this study included 40 patients, who underwent assessment with recent MRI sequences DWI, DCE MRI, T2-weighted imaging, steady-state free precession (SSFP) imaging and T1 in-phase/out-phase imaging. Results: Half of the studied patients were females. Age ranged from 30 to 67 years with mean 48.825 years. Forty percent of patients had right-side lesions while $22.5 \%$ had left side lesions. On HPE of the studied patients, $52.5 \%$ had malignant growth while the remaining $47.5 \%$ had benign lesions. About 23\% had HCC. One fifth had hemorrhagic cyst and the same percentage had
\end{abstract} metastatic lesion. Adenoma was present in $7.5 \%$, There is statistically significant relation between nature of foci and ADC value (significantly higher in benign lesions). ROC curve of ADC in diagnosis of benign nature of focal hepatic masses among the studied patients; area under curve 0.945, $(\mathrm{p}<0.05)$. Conclusion: diffusion-weighted MRI sequence with quantitative ADC measurements should be used as an additional sequence to supplement conventional MRI protocol studies for proper detection (using low $b$ values) and characterization (using high $b$ values) of focal liver lesions

Keywords: magnetic resonance; MRI; focal; hepatic 


\section{Introduction}

Primary hepatic malignancies may arise from any cell types within the liver. They are broadly classified as epithelial or mesenchymal based on their cell of origin. Of all hepatic neoplasms (benign and malignant), $93 \%$ are malignant epithelial neoplasms, and $1 \%$ are mesenchymal malignancies. The remaining $6 \%$ are benign neoplasms (1).

Magnetic resonance imaging (MRI) has more advantages than ultrasound, computed tomography (CT), positron emission tomography ( PET), or any other imaging modality in diagnosing focal hepatic masses. With a combination of basic $\mathrm{T} 1$ and $\mathrm{T} 2$ weighted sequences, diffusion weighted imaging (DWI), dynamic contrast-enhanced (DCE) MRI and T1 in-phase/out-phase, most liver lesions can be adequately diagnosed. Benign lesions, as cyst, hemangioma, extramedullary hematopoiesis, inflammatory pseudotumors, bile duct hamartomas, abscesses, focal nodular hyperplasia $(\mathrm{FNH})$ or adenoma, can be distinguished from malignant lesions. In a non-cirrhotic liver, the most common malignant lesions are metastases which may be hypovascular or hypervascular. In the cirrhotic liver hepatocellular carcinoma is of considerable importance (2).
With advances in hardware and coil systems, DW MR imaging can now be applied to liver imaging with improved image quality. DW MR imaging enables qualitative and quantitative assessment of tissue diffusability (apparent diffusion coefficient) without the use of gadolinium chelates, which makes it a highly attractive technique, particularly in patients with severe renal dysfunction (3).

The recent developments in MRI sequences for the upper abdomen comprise unenhanced diffusion-weighted imaging, and DCE MRI. DWI allows improved detection of small $(<10 \mathrm{~mm})$ focal liver lesions and useful as a road map sequence. DCE enables MRI of the liver with high temporal and spatial resolution and full liver coverage and improves evaluation of focal liver lesions, providing multiple arterial and venous phases. DCE MRI has potential benefits in terms of detection, characterization of hepatic focal lesions (4).

The primary modalities for liver imaging are US and CT, recent studies have suggested that CE-MRI is the most sensitive method for detecting small focal liver lesions in particular. One of the advantages of MRI in liver imaging is the better soft tissue contrast, which allows better detection and 
characterization of benign and malignant focal liver lesions. The development of liver-specific MRI contrast agents has further improved the diagnostic yield of MRI in lesion detection and characterization (5).

The aim of this work was to study recent and advanced magnetic resonance imaging techniques in the evaluation of focal hepatic lesions.

\section{Patients and methods}

This prospective study included 40 patients, conducted at private diagnostic imaging centers equipped with 1.5 tesla (MRI) magnets. During the period from May 2019 to May 2020.

Before starting the research both informed participant consent and Benha University ethical committee approval were taken.

\section{Inclusion criteria:}

Patient known to have focal hepatic lesions detected by US, MSCT or biopsied.

\section{Exclusion criteria:}

- Unstable

$$
\text { clinical }
$$
status, contraindications to MR imaging; claustrophobia, patients with pace maker or metal implants and patient with risk of nephrogenic systemic fibrosis.

- Patients with poor general condition who cannot tolerate:
- Breath holding for 13 seconds.

- Cannot keep still for the whole exam time.

- Claustrophobic patients.

- Patients with Pacemaker.

All patients were exposed for clinical examination, U/S and/or MSCT. MRI was done after written or verbal consent taken from the patients. Patients were fasting for at least 4 hours and cannulated before examination.

All patients underwent assessment with recent MRI sequences DWI, DCE MRI, T2weighted imaging, steady-state free precession (SSFP) imaging and T1 inphase/out-phase imaging.

The final diagnosis was reached according to Standard of reference (SOR): For lesion characterization, all imaging results were refined against a predefined SOR. A range of procedures were identified as valid "gold standards" for the characterization of lesions.

The ethically accepted and valid SOR for the lesions of HCC were the characteristic enhancement pattern on CT and/or MRI and elevated alpha fetoprotein. Simple hepatic cysts were diagnosed with typical US and MRI findings. Hemangiomas were diagnosed easily with their characteristic US and/or MRI findings and unchanged mass dimensions at following radiological 
procedures. Based on ethical considerations, we didn't expect to get SOR result for every lesion detected in patients with multiple lesions.

\section{The statistical methods:}

Data were statistically described in terms of mean \pm standard deviation ( \pm SD), and range, or frequencies (number of cases) and percentages when appropriate. Accuracy was represented using the terms sensitivity and specificity.. All statistical calculations were done using computer program SPSS (Statistical Package for the Social Science; SPSS Inc., Chicago, IL, USA) version 15 for Microsoft Windows.

\section{Results}

Half of the studied patients were females. Age ranged from 30 to 67 years with mean 48.825 years. Forty percent of patients had right-side lesions while $22.5 \%$ had left side lesions (Table 1).

Regarding echogenicity of foci detected by US, one quarter of patients had hypoechoic lesion, $47.5 \%$ had heterogeneous echogenicity, $17.5 \%$ had hyperechoic lesion while $10 \%$ had anechoic lesions (fig. 1).

On HPE of the studied patients, 52.5\% had malignant growth while the remaining 47.5\% had benign lesions. About 23\% had HCC. One fifth had hemorrhagic cyst and the same percentage had metastatic lesion. Adenoma was present in $7.5 \%$ (fig. 2).
There is statistically significant relation between nature of foci and ADC value (significantly higher in benign lesions) (Table 2).

There is statistically significant relation between pathological type of malignancy and ADC value. On LSD comparison, the difference is significant between HCC and metastasis groups (Table 3 ).

ROC curve was done to assess performance of ADC in diagnosis of benign nature of focal hepatic masses among the studied patients; The best cutoff of ADC in prediction of benign nature of foci is $\geq 1.385$ $\times 10-3 \mathrm{~mm} 2 / \mathrm{sec}$ with area under curve 0.945 , at sensitivity $100 \%$, specificity 90.5\%, positive predictive value $90.5 \%$, negative predictive value $100 \%$ and accuracy $95 \%(\mathrm{p}<0.05)$, figure 3

\section{Case presentation (fig. 3)}

Clinical history: A 38-year old female patient complaining of upper abdominal pain and constipation.

Abdominal US revealed an irregular hyperechoic hepatic focal lesion with central hypoechogenicity seen within segment VI.

MRI of the liver showed that the right hepatic lobe segment VI is the seat of an irregular well-defined focal lesion eliciting low T1 and high T2 signals with central area of breaking down. After contrast injection the lesion showed peripheral early arterial 
enhancement with centripetal pattern of enhancement in the portal and delayed phases.

On DWIs: The lesion appeared bright and remained bright on increasing the $b$ value and in ADC map the lesion remained bright.
ADC values: of the peripheral part of the lesion is $1.69 \times 10-3 \mathrm{~mm} 2 / \mathrm{sec}$ and the central breaking down is $2.7 \times 10-3 \mathrm{~mm} 2 / \mathrm{sec}$.

Diagnosis: Hemangioma

Table (1) Distribution of the studied patients according to site of lesion:

\begin{tabular}{lll}
\hline & $\mathbf{N}=\mathbf{4 0}$ & $\mathbf{\%}$ \\
\hline Right lobe & 16 & 40 \\
Left lobe & 9 & 22.5 \\
Both lobes & 3 & 7.5 \\
IV & 3 & 7.5 \\
VI & 9 & 22.5 \\
\hline
\end{tabular}

Table (2) Relation between ADC and nature of lesion among the studied patients:

\begin{tabular}{lllll}
\hline$\times \mathbf{1 0}^{\mathbf{- 3}} \mathbf{~ m m}^{\mathbf{2}} / \mathbf{s e c}$ & $\begin{array}{l}\text { Nature } \\
\text { Benign }\end{array}$ & Malignant & $\begin{array}{l}\text { Test } \\
\mathbf{t}\end{array}$ & $\mathbf{p}$ \\
ADC: & & & & \\
Mean SD & $1.778 \pm$ & $1.18 \pm 0.264$ & 6.337 & $<0.001^{* *}$ \\
Range & 0.332 & & & \\
\hline
\end{tabular}

$* * \mathrm{p} \leq 0.001$ is statistically highly significant $\mathrm{t}$ Independent sample $\mathrm{t}$ test

Table (3) Relation between ADC and nature of lesion among the studied patients:

\begin{tabular}{llllll}
\hline & $\begin{array}{l}\text { Types of malignancy } \\
\text { Cholangiocarcinoma }\end{array}$ & HCC & Metastasis & Test & p \\
\hline ADC: & & $1.346 \pm 0.29$ & $0.978 \pm 0.084$ & 6.413 & $0.008^{*}$ \\
Mean SD & $1.21 \pm 0.173$ & $1.05-1.83$ & $0.9-1.07$ & & \\
Range & $1.06-1.36$ & $\mathrm{P}_{2} 0.002^{*}$ & $\mathrm{P}_{3} 0.091$ & & \\
& $\mathrm{P}_{1} 0.302$ & & & \\
\hline
\end{tabular}

${ }^{*} \mathrm{p}<0.05$ is statistically significant $\mathrm{F}$ One Way ANOVA test $\mathrm{p} 1$ the difference between cholangiocarcinoma and HCC groups P2 the difference between metastasis and HCC groups P3 the difference between cholangiocarcinoma and metastasis groups

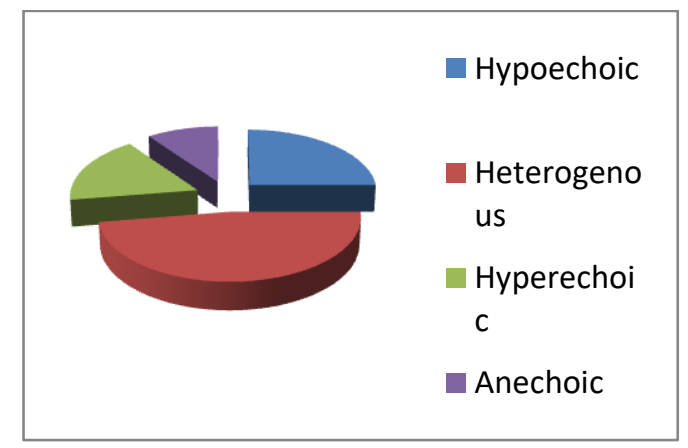

Figure (1) Pie chart showing distribution of the studied lesions according to echogenicity 


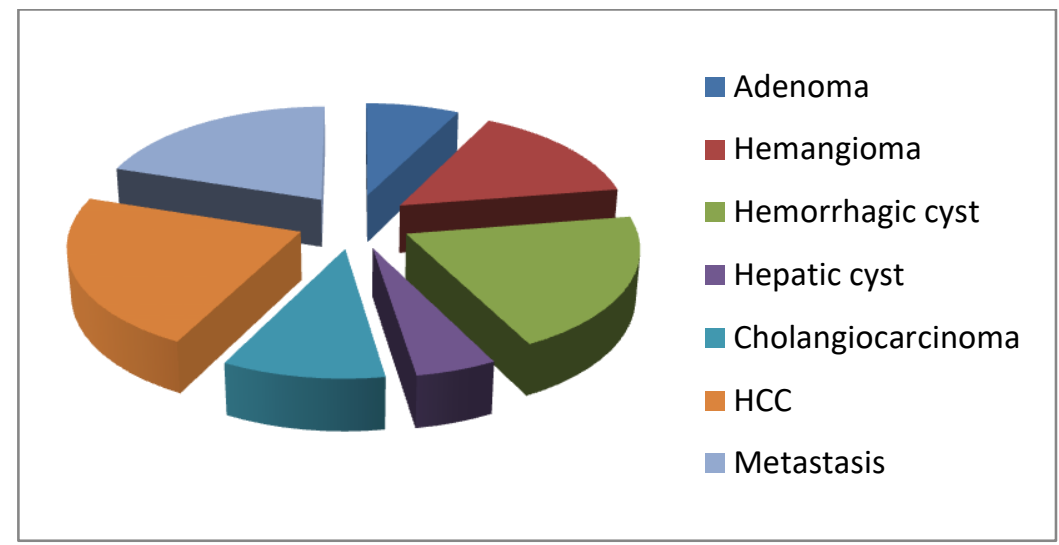

Figure (2) Pie chart showing distribution of the studied lesions according to pathological type

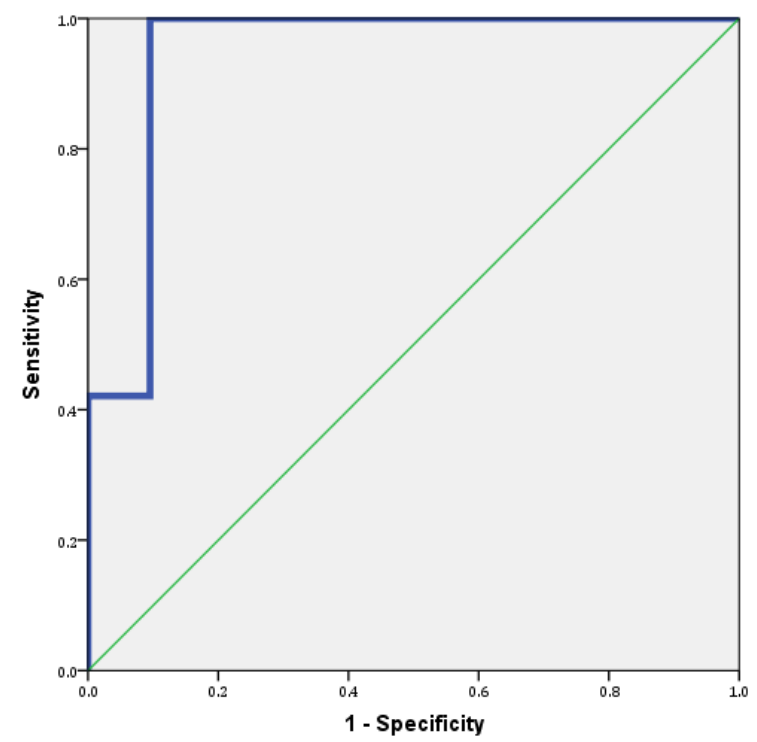

Figure (3) ROC curve showing performance of ADC in diagnosis of benign nature of focal hepatic masses among the studied patients

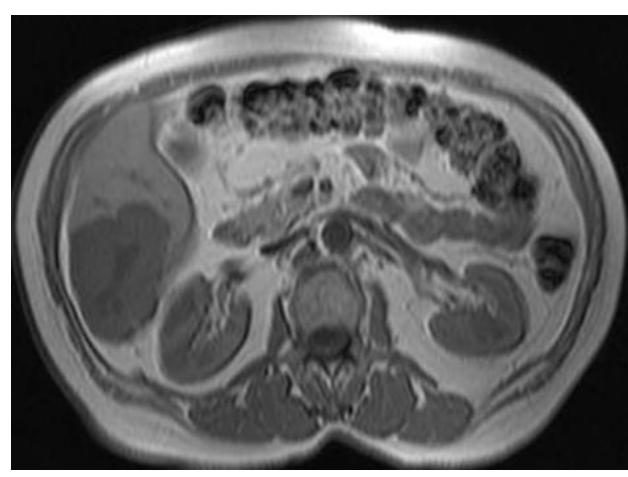

Figure 3a Axial T1WI

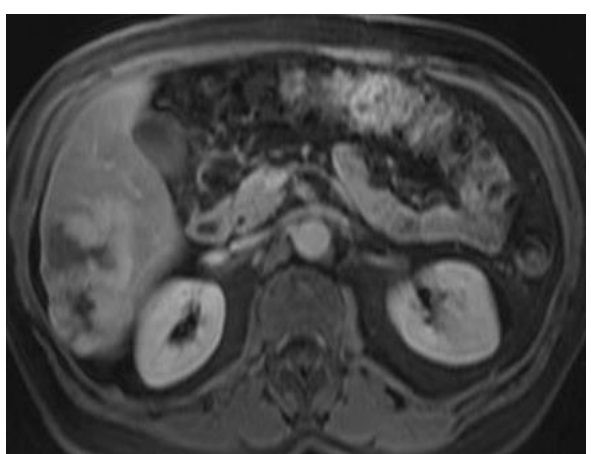

Figure 3b Axial T2WI

Fig 3 a and 3b Axial T1 and T2 respectively, showing an irregular shaped focal lesion implicating the postero-inferior segment of the right hepatic lobe (segment VI), eliciting hypointense T1 and hyperintense $\mathrm{T} 2$ signal intensities with central area of breaking down witheliciting fluid signal intensity. 


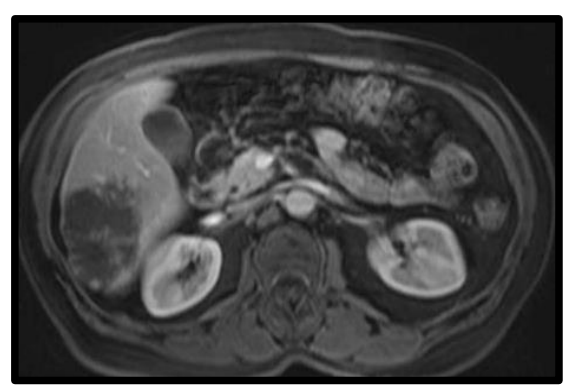

Figure 3c Axial post contrast T1 arterial delayed

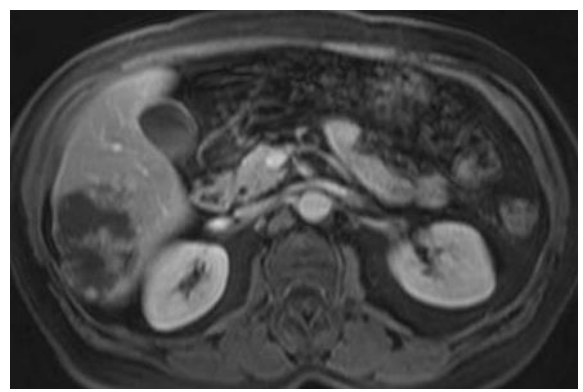

Figure 4d Axial post contrast T1 portal

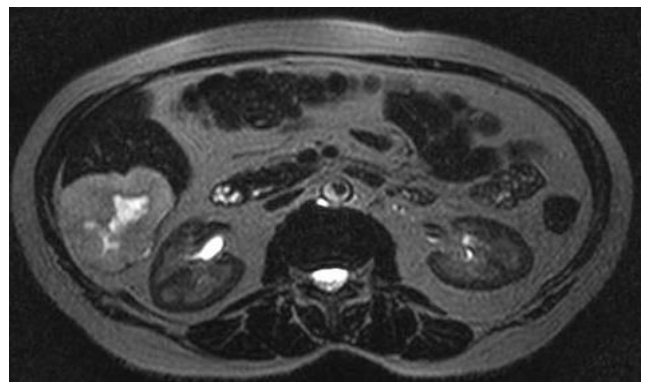

Figure 3e Axial post contrast T1

Fig 3c, Fig 3d and Fig 3e Axial T1 post contrast Arterial, porto-venous and delayed phases respectively, showing post contrast peripheral marginal enhancement in the arterial phase with centripetal pattern of enhancement in the portovenous and delayed phase giving a closed iris appearance

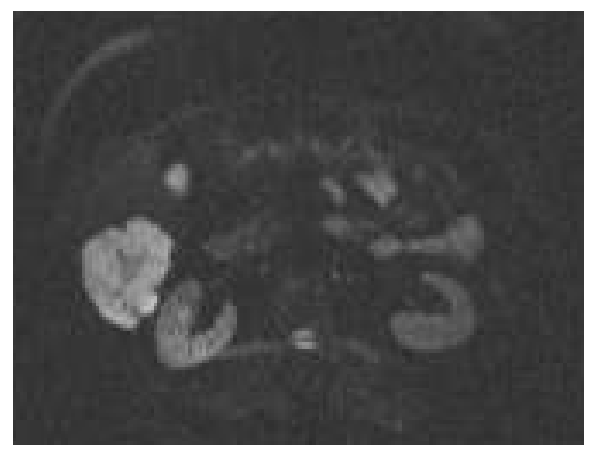

Figure 3f Axial DWI b500

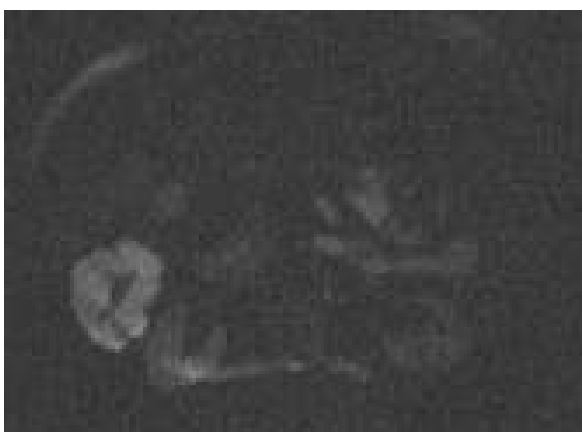

Figure 3g Axial DWI b1000

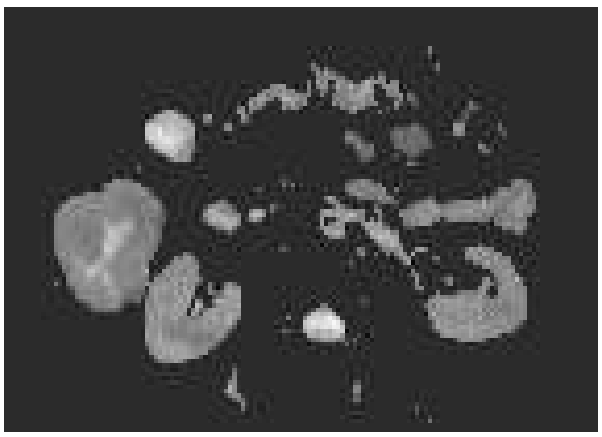

Figure 3h Axial ADC map

Fig 3f and Fig 3g Axial DWIs b500 and b1000 respectively and Fig 3h ADC map, showing a bright right hepatic lobe lesion which decreases in brightness as we increase the value due to masking of the T2 shine through effect suggesting its benign nature which was also confirmed by being intensely bright in the ADC map.

\section{Discussion}

The current study was conducted including forty patients, 20 males and 20 females, with age ranging from 30 to 67 years and mean age of 48.825 years, which means hepatic lesions were more predominant near age 50 years which was in line with another study (6), which included 382 patients with age ranging from 15-88 years and mean age was 59.2 years. and in another study done in 2010 (7), including 26 patients with age ranging from $30-73$ years and mean age was 51.5 years. This study was conducted with high $b$ value $(800 \mathrm{sec} / \mathrm{mm} 2)$ to overcome the effect of capillary perfusion and water diffusion in extracellular extravascular space, as high $b$ value will result in the reduction of signal from moving protons in the bile ducts, cysts, vessels, and fluid in the bowel. This will result in an increased contrast between the lesion and liver. 
Furthermore, the differences in the relative contrast ratio between malignant and benign lesions were increased with a high $b$ value. This was similar to the $b$ value in another study (8).

In our study, small lesions were effectively detected on diffusion weighted images, where lesions as small as $1.15 \mathrm{~cm}$ were clearly depicted, thus making DW MRI a useful tool for detection of small focal hepatic lesions even without contrast injection.

In the current study cysts and hemangiomas showed facilitated diffusion whereas solid lesions like metastasis and HCC showed restricted diffusion. On HPE of the studied patients, $52.5 \%$ had malignant growth while the remaining $47.5 \%$ had benign lesions. About 23\% had HCC, one fifth had hemorrhagic cyst and the same percentage had metastatic lesion. Adenoma was present in $7.5 \%$. This data is similar to another study (9), that stated that cellular tissues, such as tumors, demonstrates restricted diffusion (high signal intensity) on higher $b$ value $(500 \mathrm{sec} / \mathrm{mm} 2)$ images and by contrast, cysts and hemangiomas show a greater degree of signal attenuation on higher $\mathrm{b}$ value diffusion images.

The absolute ADC values of the different types of lesions were not similar, which is probably due to differences in techniques 75 applied (b value, breath measurement methods, and mathematical technique applied). This finding was also stated by another researchers (10), they stated that in spite there are an increasing number of studies dealing with quantitative measurements of ADC in liver lesions, there are many discrepancies in the reported ADC values where there is no cut-off value for ADC values in normal parenchyma, benign and malignant lesions.

This is often associated with many technical parameters such as the use of respiratorytriggered versus breath-hold diffusionweighted protocol and significantly $b$ value as high $b$ value results in low ADC value and vice versa. ADC of the studied lesion ranged from 0.9 to $2.54 \times 10-3 \mathrm{~mm} 2 / \mathrm{sec}$ with a mean of $1.464 \times 10-3 \mathrm{~mm} 2 / \mathrm{sec}$. Miller et al., (6) stated that mean ADC value was $3.40 \pm 0.48 \times 10-3 \mathrm{~mm} 2 / \mathrm{sec}$.

In our study, there is statistically significant relation of ADC value with nature of foci (significantly higher in benign lesions) and pathological type of malignancy (the difference is significant between HCC and metastasis groups).

Another researchers (6) stated that the lowest $\mathrm{ADC}$ values belonged to metastases with a mean $\mathrm{ADC}$ value of $1.03 \pm 0.17 \times 10-3$ $\mathrm{mm} 2 / \mathrm{sec}$. The ADC values of benign hepatic lesions were significantly higher 
than that of malignant hepatic tumors, with a $\mathrm{P}$ value $<0.05$. They found considerable overlap of solid benign and solid malignant lesions and stated that there were no statistically significant difference in ADC values between hepatic adenomas, FNH, hepatic metastases and HCC.

Another researchers (7) stated that the mean ADC value obtained for benign lesions differed significantly from the average for malignant lesions with a $\mathrm{p}$ value $<0.05$. and another study (11) stated that the lowest ADC values belonged to metastases with a mean ADC value of $1.05 \pm 0.22 \times 10-3$ $\mathrm{mm} 2 / \mathrm{sec}$. The mean ADC values of benign lesions were higher than malignant lesions and these differences were statistically significant for all 3 diffusion gradients with $P$ values of $<0.0023,<0.0001$, and $<0.0001$. In our study, the best cutoff of ADC in prediction of benign nature of foci is $\geq 1.385$ $\times 10-3 \mathrm{~mm} 2 / \mathrm{sec}$ with area under curve of 0.945 , at sensitivity of $100 \%$, specificity of $90.5 \%$, positive predictive value of $90.5 \%$, negative predictive value of $100 \%$ and accuracy of $95 \%(\mathrm{p}<0.05)$.

\section{Conclusion}

In our study, we concluded that diffusionweighted MRI sequence with quantitative ADC measurements should be used as an additional sequence to supplement conventional MRI protocol studies for proper detection (using low $\mathrm{b}$ values) and characterization (using high $b$ values) of focal liver lesions. The ADC values of benign lesions are significantly higher than those of malignant lesions, with variable degrees of overlap between the pathological entities; in our study, the cut-off value for prediction of benign nature of foci was 1.385 .

\section{References}

1. Yin $\mathrm{Z}$, Jiang $\mathrm{K}$, Li $\mathrm{R}$, Dong $\mathrm{C}$, Wang $\mathrm{L}$. Multipotent mesenchymal stromal cells play critical roles in hepatocellular carcinoma initiation, progression and therapy. Mol Cancer. 2018;17(1):178.

2. Albiin N. MRI of focal liver lesions. Curr Med Imaging. 2012;8(2):107-16.

3. Bharwani N, Koh DM. Diffusion-weighted imaging of the liver: an update. Cancer imaging. 2013;13(2):171.

4. Coenegrachts K. Magnetic resonance imaging of the liver: New imaging strategies for evaluating focal liver lesions. World J Radiol. 2009;1(1):72.

5. Lee JM, Yoon J-H, Joo I, Woo HS. Recent advances in CT and MR imaging for evaluation of hepatocellular carcinoma. Liver Cancer. 2012;1(1):22-40.

6. Miller FH, Hammond N, Siddiqi AJ, Shroff S, Khatri G, Wang Y, et al. Utility of diffusionweighted MRI in distinguishing benign and malignant hepatic lesions. J Magn Reson Imaging. 2010;32(1):138-47. 
7. Vergara ML, Fernández M, Pereira R. Diffusionweighted MRI characterization of solid liver lesions. Rev Chil Radiol. 2010;16(1):510.

8. Hosny IA. Diffusion MRI of focal liver lesions. PJR. 2016;20(1).

9. Taouli B, Koh D-M. Diffusion-weighted MR imaging of the liver. Radiology. 2010;254(1):47-66.
10. Kele PG, van der Jagt EJ. Diffusion weighted imaging in the liver. World J Gastroenterol WJG. 2010;16(13):1567.

11. Onur MR, Çiçekçi M, Kayalı A, Poyraz AK, Kocakoç E. The role of ADC measurement in differential diagnosis of focal hepatic lesions. Eur $\mathbf{J}$ Radiol. 2012;81(3):e171-6.

To cite this article: Ahmed F. Youssef, Amany M. Elkharbotly, Hamada M. Khater, Magdy A. Awad. State of The Art of Magnetic Resonance Imaging (MRI) in Evaluation of Focal Hepatic Lesions. BMFJ 2022; 39 (Radiology): 68-77. DOI: 10.21608/bmfj.2021.49593.1342 
Benha medical journal, vol. 39, special issue (radiology), 2022 
